\title{
Recognition of Facial Expressions for Images using Neural Network
}

\author{
Shubhangi Giripunje \\ Research Scholar, \\ Dept.of Electronics Engg., GHRCE, \\ Nagpur, India
}

\author{
Preeti Bajaj \\ Senior IEEE Member, Professor, Dept.of \\ Electronics, G.H.Raisoni College of Engineering, \\ Nagpur, India
}

\begin{abstract}
Globally terrorism continues to destroy the lives of people. To identify the terrorist amongst the other people is very difficult rather impossible. This exploratory study aims at investigating the effects of terrorism to recognize emotions. The current paper presents a view-based approach to the representation and recognition of human facial expression. In the designing of facial expression recognition (FER) system, Authors can take advantage of the resources and developed the algorithms for the system. The system divides into 3 modules, i.e. Preprocessing, Feature Extraction and Classification. The basis of the representation is a temporal template where the features are used typically based on local spatial position or displacement of specific points and regions of the face. In this paper, five different facial expressions were considered. Extracting the features, firstly logarithmic Gabor filters were applied. Then the Optimal subsets of features were selected for each expression. The classification tasks were performed using the Neural Network. Secondly, this study indicates that the YALE database contains expressers that expressed expressions.
\end{abstract}

\section{Keywords}

Keywords- Terrorism, facial expression, emotions, Neural Network

\section{INTRODUCTION}

Globally terrorism continues to destroy the lives of people to identify the terrorist in public places or even amongst few is very difficult. If we can succeed in identification of correct emotional state of the human being and then train such system so as to identify expressions then probably terrorists will be identified easily even at the crowded place. To determine emotional state of any individual it is required to know his or her facial expressions. Facial Expression is a bodily state involving various physical structures; it is gross or finegrained behavior, and it occurs in particular situations. Any empirical research on facial expression involves only some part of the broad meaning of the term. After over a century of research, facial expression of terrorists still do debate upon what kind of facial expressions are and how they are communicated. Facial expressions comprise more than its outward physical expression; it also consists of internal feelings and thoughts, as well as other internal processes of which the person having the emotion may not be aware [1]. In worldwide, many researchers have studied the automatic facial expression recognition which facilitate a more intelligent and natural human machine interface of new multimedia product. Several methods and algorithms have been proposed for facial expression analysis from both static images and image sequences.
In 26/11 Mumbai terror attack, terrorists were stayed in Mumbai for two months before attack. To recognize them as a terrorist is public place is very difficult for local public and police also. Just to looking towards the attack on September 11 , it is immensely feeling about this technology which should be more widely used amongst researchers, advocates, designers, citizen groups, political leaders and manufacturers and if it used then we have to give more emphasis on the protection of the privacy of public. Taking into consideration all these important and sensitive issues, some airports are considering Face recognition cameras as a security measure.

In this paper authors have focused on the recognition of facial expression from single digital images with emphasis on the feature extraction. This paper is organized in three sections. Section I describes Preprocessing, section II narrates the Feature extraction by Gabor filter and section III includes the results of terrorist Facial Expression recognition using Neural Network.

\section{METHODOLOGIES}

The facial expression recognition problem has lately undergone various approaches that may be divided in two main categories: static and motion dependent. In static approaches, recognition of a facial expression is performed using a single image of a face. Motion dependent approaches extract temporal information by using at least two instances of a face in the same emotional state [5]. In the static approach the performance and generalization capabilities of different low-dimensional representations for facial emotion classification from static face images showing happy, angry, sad, and neutral expressions is used. Three general strategies are compared: The first approach consist the average face as a generic template using for each face and classifies the facial expression of each face with comparison of the best matching template. The second strategy uses a multi-layered perception trained with the back propagation of error algorithm on a subset of all facial expressions and subsequently tested on unseen face images. The third approach introduces a preprocessing step prior to the learning of an internal representation by the perception. The feature extraction stage computes the oriented response to six odd-symmetric and six even-symmetric Gabor-filters has been used in different way by different researchers.

Many researchers have interest to recognize facial expression and use human computer interactions information. Number of automatic methods developed to recognize facial expressions in images [11-14]. 


\section{IMPLIMENTATIONS}

The complete work is based on the assumption that if continuous video of these terrorists is recorded wherein their facial expressions are being captured. It is noticed that only one expression which leads to anger is detected as shown in figure.1, wherein if normal person's video is captured, it will be mix expression which is going to be detected as shown in figure.2.
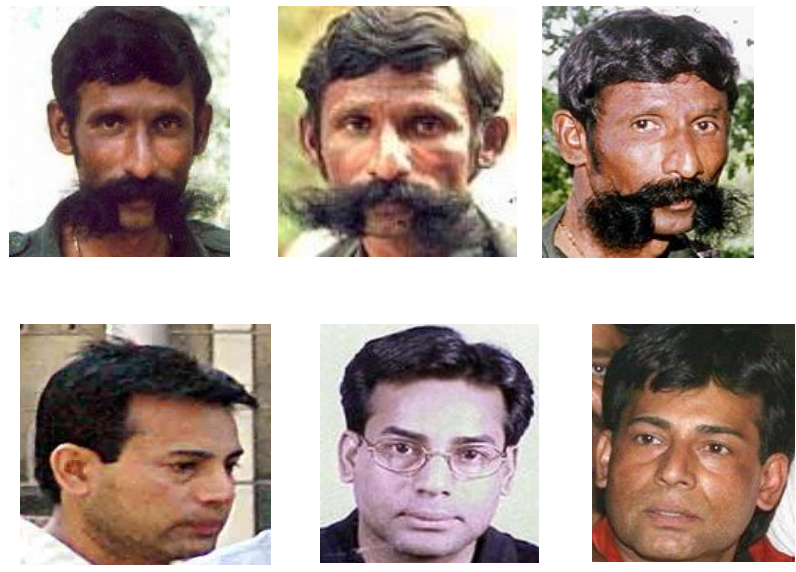

Fig1. Terrorist expression
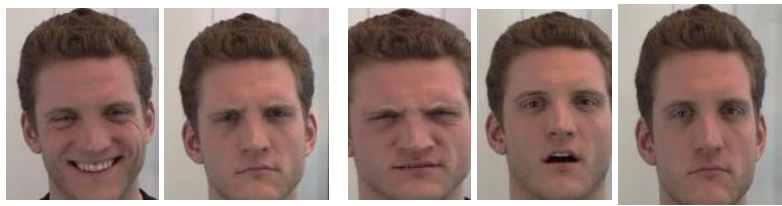

Fig2. Mixed expressions for normal persons from YALE database

\subsection{Template Matching}

In the automatic facial feature extraction technique, the use of template prototypes is portrayed on the 2-D space in grayscale format. This technique uses correlation as basic tools for comparing the template with the part of the image. To investigate the recognition and generalization performance of the various methods several computer experiments were undertaken. For extraction of textured image features the Gabor Filters is motivated by various factors. The Gabor filters can be considered as scale tunable edge and orientation and line (bar) detectors, and the statistic of these micro features in a given region is often used to characterize the underlying texture information. Gabor features have been used in several image analysis applications including textures classification and segmentation, image recognition, image registration and motion tracking.

\subsection{Gaussian Smoothing}

The Gaussian smoothing operator is a convolution operator that is used to blur the images and to remove the details and noise. It is same as a mean filter, but it represents the shape of a Gaussian ('bell-shaped') hump by using a different kernel that. The result of Gaussian smoothing is to blur an image, similarly by the mean filter. For implementation of image smoothing the simple, easy and instinctive method is Mean filter which can reduced the intensity variation between the neighbor pixels. It is often used to reduce noise in images.

The standard deviation of the Gaussian is produced the degree of smoothing. The Gaussian outputs a "weighted average" of each pixels neighborhood, with the average weighted more towards the value of the central pixels. This is in contrast to the mean filters uniformly weighted average. A gentler smoothing provides by Gaussian who preserves edges better than a similarly sized mean filter.

The Gaussian Smooth color image is then has to be converted into YCBCR image, which converts the RGB values to the YCBCR color image. Every row of YCBCR consist the same color to the particular row in RGB colormap. Then extracting the luminance $(\mathrm{Y})$ column gives the corresponding grayscale image of the given RGB image

\subsection{Primary Facial Expressions}

A most effective system developed to recognize a small set of prototypic emotional expressions. The most widely-used set is perhaps human universal facial expressions of emotion which consists of five basic expression categories that have been shown to be recognizable across cultures. All the facial expressions have been recognized in human beings from different backgrounds, and they have been noticed even from each faces even though they are deaf and blind. In Earlier years, facial expression analysis was essentially a research topic for psychologists. However, now a day's image processing and pattern recognition have motivated significant research activities on automatic facial expression recognition. Basic facial expressions, shown in Fig. 3, are happiness, surprise, anger, sadness and neutral, [YALE database].
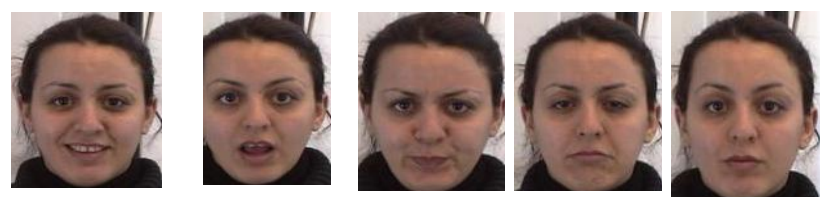

Fig 3.Basic facial expressions phenotypes

In the Fig.3 (a) shows the facial expression "Happiness" in which the eyebrows are relaxed. The corners of the mouth is pulled back headed back the ears and mouth is open. Fig.3 (b) shows the facial expression "Surprise" in which the eyebrows are raised. The upper eyelids are wide open, the lower relaxed. The jaw is opened. In the Fig.3 (c) shows the facial expression "Anger" in which the inner eyebrows are pulled downward and together. The eyes are wide open. The lips are tightly closed on each other. In the Fig.3 (d) shows the facial expression "Sadness" in which the inner eyebrows are bent upward. The eyes are slightly closed. The mouth is relaxed. In the Fig.3 (e) shows the facial expression "Neutral" in which all face muscles are relaxed. Eyelids are tangent to the iris. The mouth is closed and lips are in contact.

\subsection{Feature Extraction}

To achieve better robustness and accuracy, before the normalized facial images are fed into classifier, they are projected into some feature spaces in which, hopefully, the faces are more separable. In this way, the image processing and classifier designing can be tacked separately. In this module, we only focus on the image processing task, so when designing the classifier we can consider it as a general recognition task and won't need to think about the image 
properties. In the training stage, feature selection is performed to determine which Gabor filters are useful, so only these features are computed in the final system In facial expression recognition developed both a reliable and fast facial features extraction algorithm, whose goals is both the localization of the different facial features (mouth, eyes, eyebrows, nose, etc) and the analysis of the emotional information that they contain (through the shape, the displacements). Facial expressions give important clues about emotions. Therefore, various techniques have been proposed to classify human affective states. The facial features are used based on local spatial position or displacement of specific points and regions of the face.

\subsection{Gabor Filtering}

The Gabor Filter extracts the textured image features. In space and frequency Gabor illustration has been shown to be finest for minimization of joint two dimensional uncertainties. The direction and edge and line detectors for scale tunable consist by these filters. To characterize the underlying texture information with the help of the statistic of these micro features in a given region. Gabor features have been used in several image analysis applications including textures classification and segmentation, image recognition, image registration and motion tracking. The neighboring region of a pixel may be described by the response of a group of Gabor filters in different frequencies and directions, which have a reference to the specific pixel. In that way, a feature vector may be formed, containing the responses of those filters.

A Gabor filter is a linear filter whose impulse response is defined by a harmonic function multiplied by a Gaussian function. Therefore, a filter bank usually consists of Gabor filters with different scales and rotations. The convolution of filters is with the signal then result is in a so-called Gabor space. This process is closely related to processes in the primary visual cortex. In the image Relations between activations for a specific spatial location are very distinctive between objects Furthermore, in order to create a space object representation the important activations can be extracted from the Gabor space. The use of Gabor filtering was deemed to be one suitable tool. As it can be mathematically deduced from the filter's form, it ensures simultaneous optimum localization in the natural space as well as in frequency space. The filter is applied both on the localized area and the template in four different spatial frequencies. According to research results reported in [7-10], Gabor wavelet decomposition is useful in facial image analysis, and it's also shown that Gabor filters with different parameters carry complementary information. Consequently, it is reasonable to use a group of Gabor functions to represent images. In the Figure 4 the feature vector is shown for the emotion "Anger" which is calculated by the Gabor filter.

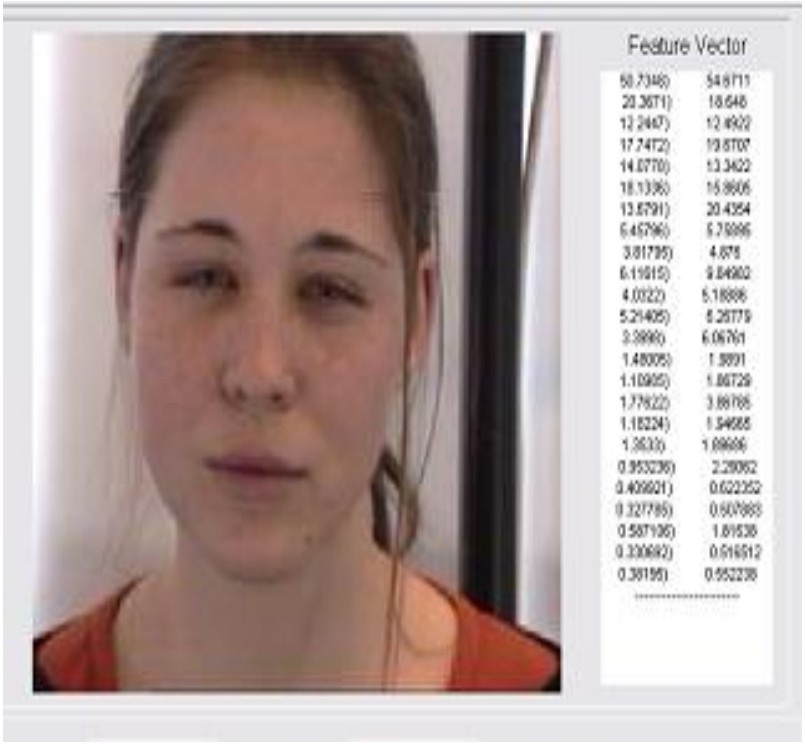

Fig.4.Facial feature vector using Gabor filter for the image of Yale database.

\section{NEURAL NETWORK -LEVENBERG- MARQUARDT ALGORITHM}

Neural Networks has the ability to derive meaning from complex and imprecise data. It can be used to extract patterns and detect trends that are much complex. In the category of information it has been given to analyze, a trained neural network can be thought of as an "expert". The LevenbergMarquardt algorithm seems to be the fastest method for training moderate-sized feed forward neural networks (up to several hundred weights). It provides a solution in numerical manner to the mathematical problem of minimizing a sum of squares of several, generally nonlinear functions that depends on a common set of parameters the minimization problem were arises in least squares curve fitting The LevenbergMarquardt algorithm (LMA) interpolates between the GaussNewton algorithm (GNA) and the method of gradient descent. With comparison, the LMA is more robust than the GNA. In many cases LMA finds a solution even if it starts very far off the final minimum. The most popular curve-fitting algorithm is LMA; in almost any software that provides a generic curvefitting tool the LMA is used; few users will ever need another curve-fitting algorithm. The flow diagram of the Facial recognition system is shown in Figure.5 


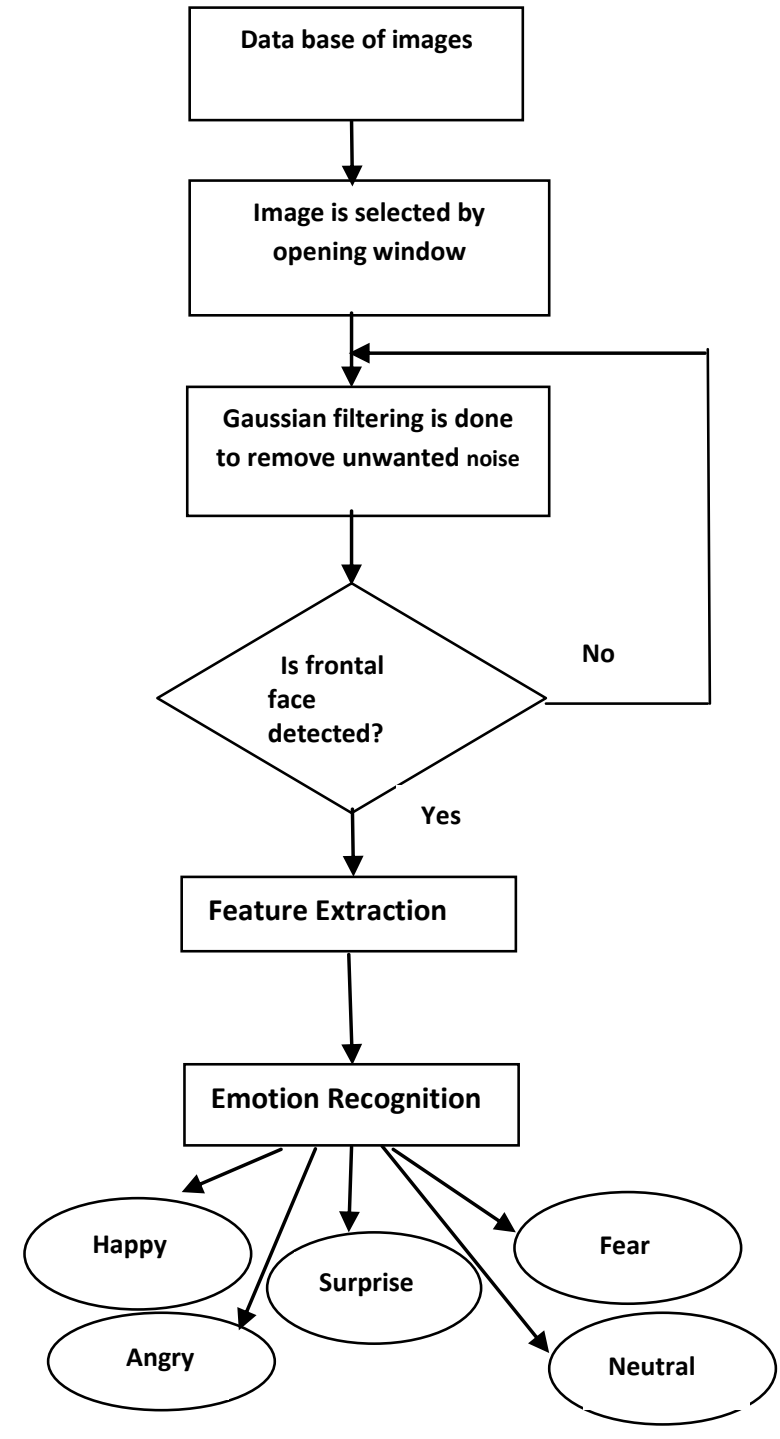

Fig 5- The flow diagram of the facial expression recognition system

\section{SIMULATION RESULTS}

The image has smoothened using Gaussian smoothing. Then it has to be converted to $\mathrm{YCbCr}$ format to eliminate chrominance and enhance luminance. After $\mathrm{YCbCr}$ image is formed image is converted to Gray Scale is shown in Figure. 6. The feature values obtained earlier are fed to the neural network, which gives a value corresponding to Facial Expression for terrorist shown in Figure.7 and Figure.8.

An analysis of the confusion matrix for YALE database (Table1) suggests that out of all the expressions, anger and neutral was the confused with all the other expressions. The database contains 125 color images in jpg format. There are 16 different people's images for different facial expressions which consist with or without glasses.

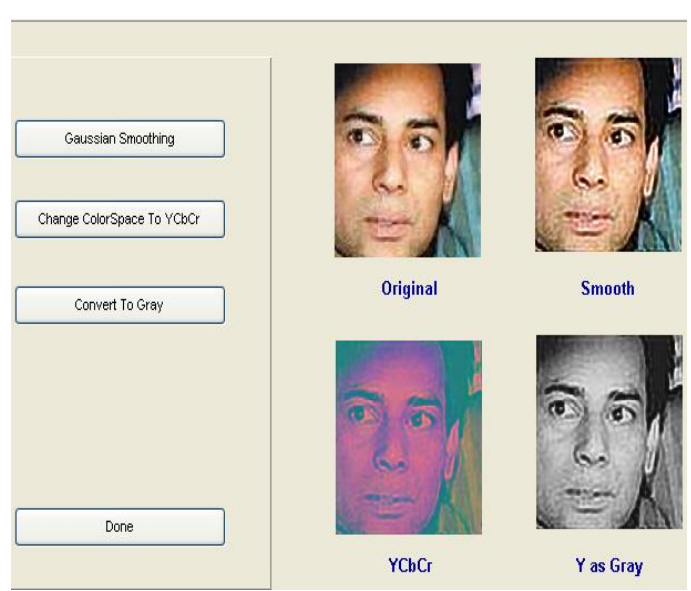

Fig6. Image Preprocessing

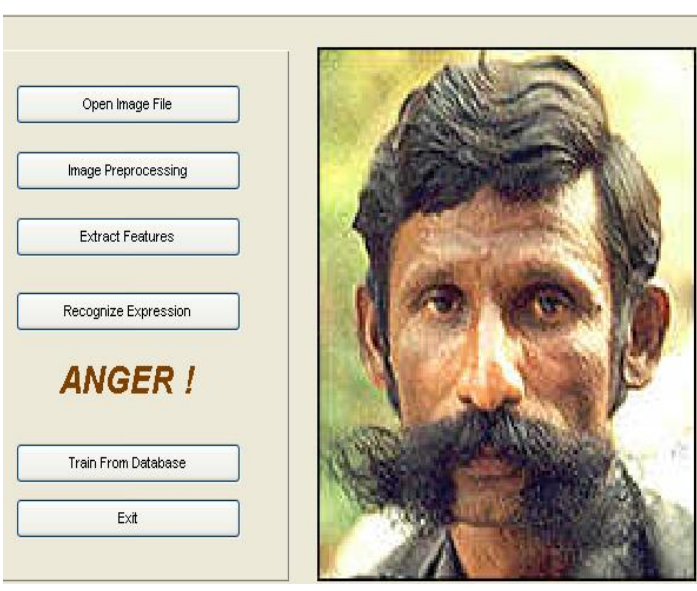

Fig7. Simulation window showing Facial expression 'Anger' of terrorist "Veerappan" has been recognized

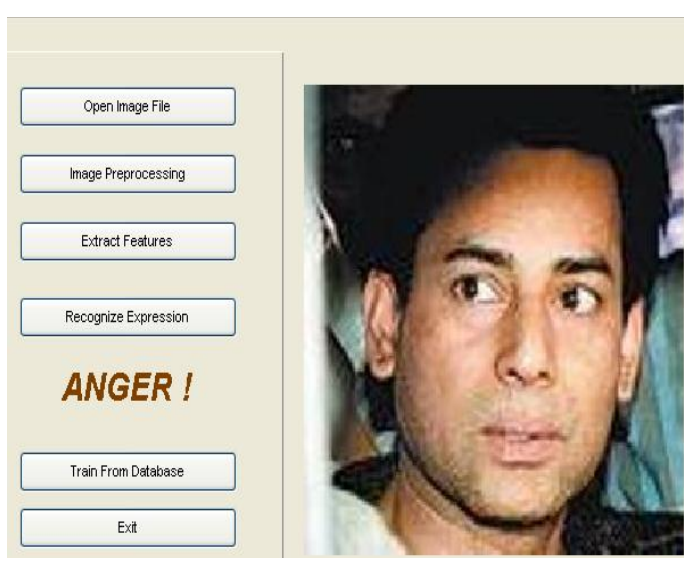

Fig8. Simulation window showing Facial expression ‘Anger' of terrorist “Abu Salem” has been recognized 


\section{CONCLUSIONS}

To identify the terrorist among the other people above approach works very well. This exploratory study aims at investigating the effects of terrorism to recognize emotions. The basis of the representation is a temporal template where the features are used typically based on local spatial position or displacement of specific points and regions of the face. In this paper, five different facial expressions were considered.
The system is working well for Yale database as well as for identifying few terrorist for whom Interpol was in search of in last decade. Although this is beginning of the research and authors are still working on various other approaches and real time data with the help of ATS department.

\section{ACKNOWLEDGMENTS}

Authors wish to thank Ajith Abraham for the valuable comments made in the initial version of the manuscript.

Table1: Confusion matrix for YALE database

\begin{tabular}{|l|l|l|l|l|l|}
\hline Expressions & Happiness & Anger & Surprise & Sad & Neutral \\
\hline Happiness & $\mathbf{9 9 . 9 9 \%}$ & $0.0 \%$ & $0.0012 \%$ & $0.0016 \%$ & $0.004 \%$ \\
\hline Anger & $0.0 \%$ & $\mathbf{9 9 . 8 2 \%}$ & $0.043 \%$ & $0.035 \%$ & $0.097 \%$ \\
\hline Surprise & $0.0 \%$ & $0.004 \%$ & $\mathbf{9 9 . 9 9 \%}$ & $0.0 \%$ & $0.0045 \%$ \\
\hline Sad & $0.0 \%$ & $0.005 \%$ & $0.0 \%$ & $\mathbf{9 9 . 9 8 \%}$ & $0.005 \%$ \\
\hline Neutral & $0.0 \%$ & $0.010 \%$ & $0.0 \%$ & $0.0 \%$ & $\mathbf{9 9 . 9 8 \%}$ \\
\hline
\end{tabular}

\section{REFERENCES}

[1] R. W. Picard, E. Vyzas, and J. Healey, "Toward machine emotional intelligence: Analysis of affective physiological state," IEEE Trans. Pattern Anal. Mach. Intell., vol. 23, no. 10, pp. 1175-1191, Oct. 2001.

[2] C.H. Messom, A Sarrafzadeh, M.J. Johnson, F. Chao "Affective state estimation from facial images using neural networks and fuzzy logic".

[3] Fasel, J. Luettin, "Automatic facial expression analysis: a survey”, Pattern Recognition, Vol. 36, 2003, pp.259-275.

[4] M. Pantic, J. M. Rothkrantz, "Automatic Analysis of Facial Expressions: The State of the Art", IEEE Trans. Pattern Analysis and Machine Intelligence, Vol. 22, 2000, pp.1424-1444

[5] Seyed Mehdi Lajevardi, Margaret Lech, "Facial Expression Recognition Using Neural Networks and Log-Gabor Filters," Digital Image Computing: Techniques and Applications, pp.77-83,2008.

[6] Christine L. Lisetti , David E. Rumelhart "Facial Expression Recognition Using a Neural Network (1998) ,In Proceedings of the 11 th International FLAIRS Conference

[7] M. S. Bartlett, P. A. Viola, T. J. Sejnowski, B. A. Golomb, J. Larsen, J. C. Hager,and P. Ekman. "Classifying facial actions" IEEE Transactions On Pattern Analysis And Machine Intelligence, Vol. 21, No. 10, October 1999
[8] Liu and H. Wechsler. Gabor feature based classification using the enhanced fisher linear discriminant model for face recognition, 2002.

[9] W. Liu and Z. Wang. 'Facial expression recognition based on fusion of multiple Gabor features' In ICPR '06: Proceedings of the 18th International Conference on Pattern Recognition, pages 536-539, Washington, DC, USA, 2006. IEEE Computer Society.

[10] P. Michel and R. E. Kaliouby. 'Real time facial expression recognition in video using support vector machines' In ICMI '03: Proceedings of the 5th international conference on Multimodal interfaces, pages 258\{264, New York, NY, USA, 2003. ACM.

[11] Ruicong Zhi, Qiuqi Ruan, "A Comparative Study on Region-Based Moments for Facial Expression Recognition,", in Congress on Image and Signal Processing, Vol. 2, pp.600-604, 2008.

[12] Irene Kotsia, Ioannis Pitas, “ Facial Expression Recognition in Image Sequences Using Geometric Deformation Features and Support Vector Machines" in IEEE Transactions on Image Processing 16(1): pp.172$187,2007$.

[13] Kakumanu.P., Nikolaos G. Bourbakis, “ A Local-Global Graph Approach for Facial Expression Recognition". ICTAI, pp 685-692,2006.

[14] Aleksic. P.S., Aggelos K. Katsaggelos. "Automatic facial expression recognition using facial animation parameters and multistream HMMs".IEEE Transactions on Information Forensics and Security 1(1): pp. 3-11, 2006. 